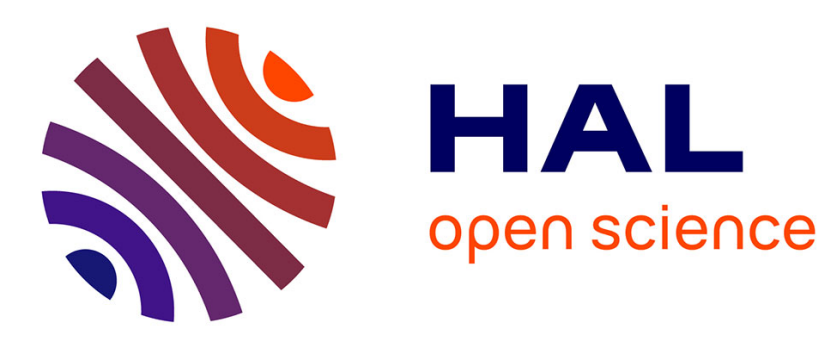

\title{
MultiScale Wavelet p-Leader based Heart Rate Variability Analysis for Survival Probability Assessment in CHF Patients
}

\author{
Herwig Wendt, Ken Kiyono, Patrice Abry, Junichiro Hayano, Eiichi \\ Watanabe, Yoshiharu Yamamoto
}

\section{To cite this version:}

Herwig Wendt, Ken Kiyono, Patrice Abry, Junichiro Hayano, Eiichi Watanabe, et al.. MultiScale Wavelet p-Leader based Heart Rate Variability Analysis for Survival Probability Assessment in CHF Patients. IEEE Engineering in Medicine and Biology Society Conference - EMBC 2014, Aug 2014, Chicago, United States. pp. 2809-2812. hal-01150339

\author{
HAL Id: hal-01150339 \\ https://hal.science/hal-01150339
}

Submitted on 11 May 2015

HAL is a multi-disciplinary open access archive for the deposit and dissemination of scientific research documents, whether they are published or not. The documents may come from teaching and research institutions in France or abroad, or from public or private research centers.
L'archive ouverte pluridisciplinaire HAL, est destinée au dépôt et à la diffusion de documents scientifiques de niveau recherche, publiés ou non, émanant des établissements d'enseignement et de recherche français ou étrangers, des laboratoires publics ou privés. 


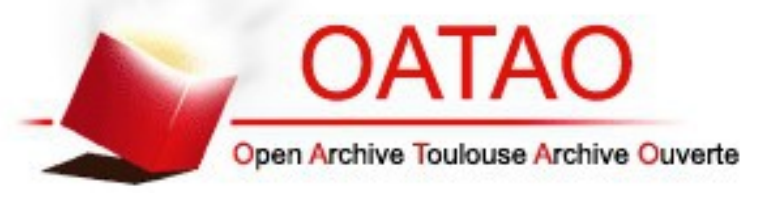

\section{Open Archive TOULOUSE Archive Ouverte (OATAO)}

OATAO is an open access repository that collects the work of Toulouse researchers and makes it freely available over the web where possible.

This is an author-deposited version published in : http://oatao.univ-toulouse.fr/ Eprints ID : 13130

To link to this article : DOI :10.1109/EMBC.2014.6944207 URL : http://dx.doi.org/10.1109/EMBC.2014.6944207

To cite this version : Wendt, Herwig and Kiyono, Ken and Abry, Patrice and Hayano, Junichiro and Watanabe, Eiichi and Yamamoto, Yoshiharu MultiScale Wavelet p-Leader based Heart Rate Variability Analysis for Survival Probability Assessment in CHF Patients. (2014) In: IEEE Engineering in Medicine and Biology Society Conference EMBC 2014, 26 August 2014 - 30 August 2014 (Chicago, United States).

Any correspondance concerning this service should be sent to the repository administrator: staff-oatao@listes-diff.inp-toulouse.fr 


\title{
MultiScale Wavelet p-Leader based Heart Rate Variability Analysis for Survival Probability Assessment in CHF Patients
}

\author{
H. Wendt ${ }^{1}$, K. Kiyono ${ }^{2}$, P. Abry ${ }^{3}$, J. Hayano ${ }^{4}$, E. Watanabe ${ }^{5}$, Y. Yamamoto ${ }^{6}$
}

\begin{abstract}
A priori discrimination of high mortality risk amongst congestive heart failure patients constitutes an important clinical stake in cardiology and involves challenging analyses of the temporal dynamics of heart rate variability (HRV). The present contribution investigates the potential of a new multifractal formalism, constructed on wavelet p-leader coefficients, to help discrimination between survivor and non survivor patients. The formalism, applied to a high quality database of 108 patients collected in a Japanese hospital, enables to assess the existence of multifractal properties amongst congestive heart failure patients and to reveal significant differences in the multiscale properties of HRV between survivor and non survivor patients, for scales ranging from approximately 60 to 250 beats.
\end{abstract}

\section{INTRODUCTION}

Congestion Heart Failure: Discriminating Survivors from Non-Survivors. Human Heart Rate Variability (HRV) is known to fluctuate in a highly irregular and complex manner that reflects the health status of patients. The analysis of HRV fluctuations can notably be used to discriminate healthy subjects from patients suffering from congestive heart failure (CHF). However, despite significant recent advances in therapy, CHF is a difficult condition to manage in clinical practice and mortality remains unacceptably high. Medical studies have consistently reported a significant decrease in mortality for patients equipped with implantable cardioverterdefibrillators or undergoing resynchronization therapy. Risk stratification for deciding a priori which patients should benefit from such treatments therefore constitutes a stake of major clinical importance. Identification of high-risk patients with CHF remains a difficult and challenging task with current available methods. It has thus recently received growing academic interest and research efforts (cf., e.g., [1]-[3]).

Related works. After the seminal contribution [4] that stimulated the massive use of spectral analysis for HRV characterization, the benefits of using alternative tools dedicated to non linear, non Gaussian and non stationary analyses were investigated (cf., e.g., [5], [6] and reference therein). In the last two decades, evidence was gathered that the temporal dynamics of HRV fluctuations are well described by fractal,

\footnotetext{
Work supported by ANR BLANC 2010 FETUSES 18535

1 CNRS, University of Toulouse, France, herwig.wendteirit.fr

2 Division of Bioengineering, Graduate School of Engineering Science, Osaka University, Toyonaka, Japan, kiyono@bpe.es.osaka-u.ac.jp

${ }^{3}$ CNRS, ENS de Lyon, France, patrice.abry@ens-lyon. fr

4 Department of Medical Education, Nagoya City University Graduate School of Medical Sciences, Japan, hayano@med.nagoya-cu.ac.jp

${ }^{5}$ Department of Cardiology, Fujita Health University School of Medicine, Toyoake, Japan, enwatan@fujita-hu.ac.jp

6 Educational Physiology Laboratory, Graduate School of Education, University of Tokyo, Japan, yamamoto@p.u-tokyo.ac.jp
}

or scaling, properties, characterized notably by $1 / f$ power spectra [7]-[10], multifractality [11], [12], or non Gaussian distributions with fat tails [13], [14]. It has been shown that the alterations of such properties can be associated with certain diseases and can thus be used as diagnostic tools [11], [15]. For instance, an increased departure from Gaussianity (in HRV increments computed on a 25 s scale) is associated with an increased cardiac mortality risk in a cohort of acute myocardial infarction (AMI), with a predictive power independent of other HRV indices [15]. Furthermore, departures from Gaussianity have been shown to be relevant in the evaluation of increased mortality risk in CHF patients [3] and compared against fractal exponent and variance in beat interarrival times reported in [2].

Goals, contributions and outline. In this context, the present contribution aims at investigating to which extent multiscale and multifractal analysis enables to help discriminating between survivor and non-survivor CHF subjects. It relies on the use of a new tool, the wavelet p-leader multifractal formalism, very recently proposed in [16]-[18] and briefly defined in Section III. This statistical analysis tool is expected to yield better estimation performance and to show significant robustness in quantifying the scaling properties of real world data [16]. It is applied to a high quality database, described in Section II, consisting of 24h HRV data for 108 CHF patients collected at Fujita Health University Hospital, Japan. Results are presented and discussed in Section IV, in terms of compared multiscale behaviors, powers of statistical tests and Kaplan-Meier Survival Curves. Conclusions are drawn in Section V, also discussing of future works.

\section{DATABASE}

Database. A cohort of $108 \mathrm{CHF}$ patients was enrolled at Fujita Health University Hospital, Japan, during years 2000-2001. The cohort was constituted of 61 male and 47 female subjects, with age ranging from 21 to 92 (average $66.1 \pm 14.8$ ) years. Of these patients, 39 (36.1\%) died within the follow-up period of $33 \pm 17$ months (range 1-59 months). Medication status before hospital discharge did not significantly differ between survivor (SV) and non-survivor (NS) patients. Clinical details for these CHF patients were previously reported in [3].

Data. For each patient, a 24-hour Holter ECG recording was collected prior to Hospital discharge. $\mathrm{R}$ peaks were extracted and thoroughly reviewed for outlier removal and detection error correction. When atrial or ventricular premature complexes occurred, they were handled by median interpolation using two successive beats. It has also been 
checked that no sustained tachyarrhythmias were present in HRV recordings. The corresponding RR inter-arrival time values, denoted as $X \equiv\left\{x_{n}, n=1, \ldots, N\right\}$, are analyzed as a time series, without resampling on a regular time grid.

\section{WAVELET P-LEADER FORMALISM}

Multifractal analysis. Multifractal analysis aims at characterizing the fluctuations along time $n$ of the local regularity of a signal $X$, classically measured by the Hölder exponent $h$, by means of the so-called multifractal spectrum $D(h)$ (cf. [19] for a theoretical introduction). $D(h)$ can be further described using multifractal parameters (e.g., the multifractal exponents described below.) It has already been massively used to analyze HRV both in adults [11], [20] and fetuses [21], [22]. Numerous declinations of multifractal formalisms (the practical counterpart of multifractal theory that actually enables to compute the multifractal parameters) were proposed and compared (cf. [20] for a review). Notably, declinations of the (discrete) wavelet transform have proven to yield some of the most relevant multifractal formalisms (cf. e.g. [22]-[24]). The present contribution relies on a new tool, referred to as the wavelet p-leader formalism, that has been theoretically introduced in [18] and recently studied in [16], [17].

Wavelet coefficients. Let $\psi$ denote the mother wavelet, characterized by a strictly positive integer $N_{\psi}$ defined as $\forall n=0, \ldots, N_{\psi}-1, \int_{\mathbb{R}} t^{k} \psi(t) d t \equiv 0$ and $\int_{\mathbb{R}} t^{N_{\psi}} \psi(t) d t \neq$ 0 and referred to as the number of vanishing moments. The ( $L^{1}$-normalized) discrete wavelet transform coefficients $d_{X}(j, k)$ of $X$ are defined as $d_{X}(j, k)=\left\langle\psi_{j, k} \mid X\right\rangle$, with $\left\{\psi_{j, k}(t)=2^{-j} \psi\left(2^{-j} t-k\right)\right\}_{(j, k) \in \mathbb{N}^{2}}$. For detailed introductions to wavelet transforms, readers are referred to e.g., [25]. Wavelet p-leaders. The p-leaders are defined as local $L^{p}$-norms of the fractional integral of order $\gamma$ of wavelet coefficient, $2^{j \gamma} d_{X}(j, k)$,

$$
L^{(p, \gamma)}(j, k)=\left(2^{j} \sum_{\lambda_{j^{\prime}, k^{\prime}} \subset 3 \lambda_{j, k}}\left|2^{j^{\prime} \gamma} d_{j^{\prime}, k^{\prime}}\right|^{p} 2^{-j^{\prime}}\right)^{1 / p},
$$

with $\lambda_{j, k}=\left[k 2^{j},(k+1) 2^{j}\right)$ and $3 \lambda_{j, k}=\bigcup_{m\{-1,0,1\}} \lambda_{j, k+m}$. The parameter $\gamma \geq 0$ must be chosen to ensure a minimal regularity constraint (cf. [16]-[18] for theoretical developments). Parameter $p$ can be freely chosen in a range $p \in$ $\left(0, p_{0}\right)$ where $p_{0}$ is implicitly defined by

$$
\eta\left(p_{0}\right)+\gamma p_{0}=0,
$$

with $1 / n_{j} \sum_{k=1}^{n_{j}}\left|d_{X}(j, k)\right|^{p} \sim K_{p} 2^{j \eta(p)}, 2^{j} \rightarrow 0$. The p-leaders enable to measure the fluctuations of the local regularity of $X$, quantified by its $p$-exponents $h_{p}$, and to infer the corresponding multifractal parameters (cf. [16]-[18] for details, beyond the scope of this contribution.)

Cumulants. The quantities used in this work for characterizing the multiscale and multifractal properties of $X$ are the cumulants $C_{m}^{(p, \gamma)}(j)=\mathrm{Cum}_{m} \ln L^{(p, \gamma)}(j, \cdot)$ of the variables $\ln L^{(p, \gamma)}(j, k)$, commonly used for multifractal analysis [24], [26]. For $X$ with perfect fractal properties (such as selfsimilar or multifractal processes), it can be shown that [26]:

$$
C_{m}^{(p, \gamma)}(j)=\nu_{m}^{(p, \gamma)}+c_{m}^{(p, \gamma)} \ln 2^{j} .
$$

When applied to exactly self-similar processes, $c_{1}^{(p, \gamma)}$ is tied to the self-similar exponent as $c_{1}^{(p, \gamma)}=H+\gamma$. It is thus generically referred to as the self-similarity exponent. Exponents $c_{m}^{(p, \gamma)}, m \geq 2$, do not depend on $\gamma$ and are directly related to the multifractal properties of data, thus referred to as multifractal exponents. Making use of (3), the exponents $c_{m}^{(p, \gamma)}$ can be estimated by linear regressions of the sample cumulants $C_{m}^{(p)}(j)$ against $\ln 2^{j}$, for $j \in\left[j_{1}, j_{2}\right]$.

\section{RESULTS AND DISCUSSIONS}

p-leaders multiscale HRV analysis. To apply the pleader multifractal formalism, parameters $\gamma$ and $p$ need to be selected. First the wavelet coefficients $d_{X}(j, k)$ are computed from the $24 \mathrm{~h}$ RR interarrival time series for each subject. Inspection shows that for most subjects Condition (2) can not be satisfied with $\gamma=0$ for any $p_{0}>0$. This provides us with a first and crucial information regarding HRV and scaling: Multifractal analysis can not in general be applied to HRV data without the a priori recourse to a fractional integration of small yet non zero order to ensure minimal regularity. The value $\gamma=0.5$ is found sufficient to ensure minimal regularity for all subjects. To simplify the characterization of the entire database, this value is used for all subjects.

With this choice for $\gamma$, the p-leaders $L^{(p, \gamma)}(j, k)$, the logcumulant functions $C_{m}^{(p, \gamma)}(j)$ and the exponents $c_{m}^{(p, \gamma)}$ can be computed for $p \in\left(0, p_{0}\right)$, where $p_{0}$ varies amongst subjects but $p_{0} \geq 4$ for all subjects. It is found that $p=1$ yields the optimal classification between SV and NS subjects. Interestingly, this low value of $p$ matches the theoretical results reported in [16], [17] showing that estimation performance generically improve with decreasing $p$. All results reported below are thus computed with parameters $(p, \gamma)=(1,0.5)$.

Furthermore, it is chosen here to analyze cumulants up to order $m=3$, i.e., $C_{1}^{(p, \gamma)}(j), C_{2}^{(p, \gamma)}(j)$ and $C_{3}^{(p, \gamma)}(j)$ corresponding respectively to the mean, the variance and (essentially) the skewness of $\ln L^{(p, \gamma)}(j, \cdot)$.

Statistical discrimination between NS and SV patients. To assess the discriminative power of p-leaders for the temporal dynamics of NS versus SV CHF patients, Wilcoxon rank-sum tests are used together with Kaplan Meier Cumulative Survival Curves (KMSC), following the methodology in [3]. KMSC curves are obtained by choosing a threshold for a chosen attribute ( $\operatorname{say} c_{1}^{(1,0.5)}$ ) and splitting the population into two groups of predicted NS and predicted SV with attributes above and below this threshold, respectively. Over the follow-up period, the population of a group decreases each time one of its members dies such that with an ideal discrimination, the population of the predicted SV class would remain constant while that of the predicted NS class would decay to zero. Differences between the decrease of the curves for the two groups are assessed by the MantelHaenszel Logrank test: Let $n_{S V}$ and $d_{S V}$ (resp., $n_{N S}$ and $d_{N S}$ ) denote the total number of subjects and the number of deaths occurring in class SV (resp., NS). Let $d=d_{S V}+d_{N S}$, $n=n_{S V}+n_{N S}$. Under the null hypothesis that the survival curves of the two classes are equal, the test statistic 

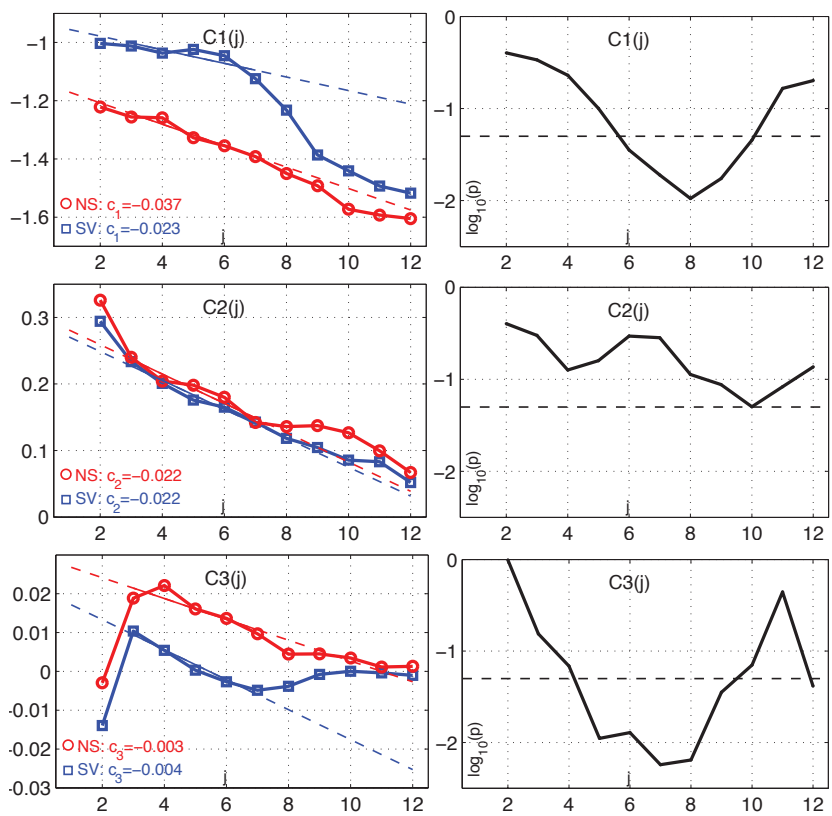

Fig. 1. Log-cumulants $C_{m}^{(1,0.5)}(j)$ as functions of the $\log$ of the scale and discrimination between SV and NS patients. Left column: $C_{m}^{(1,0.5)}(j)$ for $m=1,2,3$ from top to bottom. Right column: $\log _{10}$ (pvalue) for each octave $j$ (a value below the threshold 1.30 (dashed horizontal black line) indicates a p-value smaller than 0.05 and thus a significative difference between SV and NS).

$Z=\frac{n^{2}(n-1)\left(d_{S V}-n_{S V} d / n\right)^{2}}{n_{S V} n_{N S} d(n-d)} \sim \chi_{1}^{2}$ follows a chi-squared distribution with 1 degree of freedom.

Furthermore, we assess classification performance in terms of Sensitivity and Specificity.

Discriminative power of multifractal properties. Fig. 1 shows log-cumulants $C_{m}^{(1,0.5)}(j)$ as functions of $\left(\log _{2}\right.$ of $)$ the analysis scale $a=2^{j}$, and calls for the following comments.

First, it can be observed that, both for NS and SV, all three log-cumulant functions $C_{m}^{(1,0.5)}(j)$ (Fig. 1, left column) display satisfactory linear behavior across scales $2^{3} \leq a \leq 2^{7}$. This indicates that HRV temporal dynamics display scaling properties across groups of 8 to 128 beats. This partially matches with previous analysis of RR interarrival time series reporting scaling in range of 10 to 1000 beats [10]. Such scaling have often been quantified via the so-called $\alpha_{2}$ scaling parameter when measured by Detrended Fluctuation Analysis (DFA) (cf. e.g., [3] and references therein). Note that the slope $c_{1}^{(1,0.5)}$ of the function $C_{1}^{(1,0.5)}(j)$ can be understood as a robust estimator for $\alpha_{2}$. Thorough theoretical and practical comparisons between DFA and p-leaders will be detailed in a forthcoming study.

Second, the fact that the slopes $c_{1}^{(1,0.5)}$ differ (Fig. 1, top left) indicates a clear difference in the self-similar temporal dynamics between SV and NS subjects. Accordingly, Fig. 2 (top right) shows that KMSC computed from $c_{1}^{(1,0.5)}$ has a moderate discriminative power, slightly below the significance level 0.05 .

Third, multifractal exponents $c_{2}^{(1,0.5)}$ and $c_{3}^{(1,0.5)}$, measured from the linear behaviors across scales of the second and third log-cumulant functions, $C_{2}^{(1,0.5)}(j)$ and $C_{3}^{(1,0.5)}(j)$
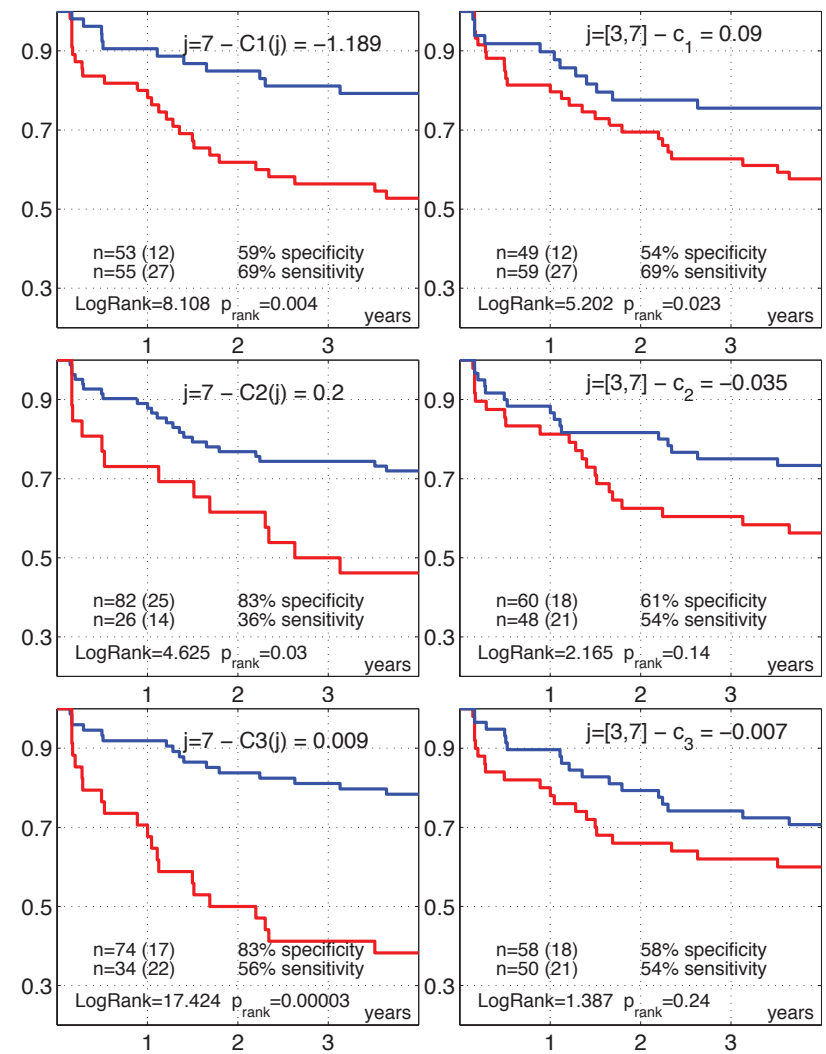

Fig. 2. Kaplan Meier Cumulative Survival Curves for the log-cumulants $C_{m}^{(1,0.5)}(j=7)$ for $m=1,2,3$ (left column, top to bottom) and for the self-similar exponent $c_{1}^{(1,0.5)}$ and multifractal exponent $\mathrm{s} c_{2}^{(1,0.5)}, c_{3}^{(1,0.5)}$.

(Fig. 1, middle and bottom left), are observed to clearly depart from 0, a strong evidence that scaling in RR interarrival HRV time series possess multifractal properties, both for SV and NS patients. The range (or power) of the fluctuations of regularity of RR interarrival HRV time series is identical for NS and SV patients. Yet, no significant change in the multifractal temporal dynamics conveyed by $c_{2}^{(1,0.5)}$ and $c_{3}^{(1,0.5)}$ is evidenced between NS and SV patients. Accordingly, KMSC computed from them (Fig. 2, middle and bottom right, respectively) show no discriminative power.

Discriminative power of multiscale properties. Multifractal analysis consists of a demanding model as it relies on the assumption that data follow (3). To alleviate such assumptions, log-cumulant functions $C_{m}^{(1,0.5)}(j)$ can be examined at each scale $a=2^{j}$ independently, regardless of whether (3) is satisfied or not. These are referred to as the multiscale properties of data (to distinguish from multifractal properties induced by (3)) and provide a measure for the non Gaussian characteristics of p-leaders at each scale $j$.

First, it is observed that the function $C_{1}^{(1,0.5)}(j)$ (Fig. 1, top right) clearly discriminates between SV and NS temporal dynamics for scales $a=2^{j}$ ranging from $2^{6} \simeq 60$ to $2^{10} \simeq 1000$ beats. Interestingly, $C_{1}^{(1,0.5)}(j)$ for NS patients is systematically below that of $\mathrm{SV}$ patients, indicating a clear decrease in the overall power (or variance) of the RR inter-arrival times HRV time series. This thus materializes 
a decrease in the overall variability of heart rate, generally considered a sign of bad health. Accordingly, KMSC computed from $C_{1}^{(1,0.5)}(j=7)$ (Fig. 2, top left) indicate a high discriminative power between NS and SV patients.

Second, the functions $C_{2}^{(1,0.5)}(j)$ (Fig. 1, middle left) show no significant change between NS and SV at any scale and the KMSC computed from $C_{2}^{(1,0.5)}(j=7)$ (Fig. 2, middle right) indicates little to no discriminative power.

Third and foremost, the third log-cumulant function (skewness type) $C_{3}^{(1,0.5)}(j)$ (Fig. 1, bottom left) is observed to significantly differ for scales ranging from $2^{4} \simeq 20$ to $2^{10} \simeq$ 1000 beats. The KMSC computed from $C_{3}^{(1,0.5)}(j=7)$ (Fig. 2, bottom left) reflect this fact and show highly significative discriminating power between NS and SV patients.

These observations confirm multifractal analysis: There exist clear yet subtle changes in multiscaling properties of RR interarrival HRV time series. Changes in $C_{1}^{(1,0.5)}(j=7)$ indicate changes in the power (or variance) of the time series themselves, a significant decrease being manifest for NS patients; The absence of change in $C_{2}^{(1,0.5)}(j=7)$ indicates that the range of the fluctuations of $\ln L^{(p, \gamma)}(j, \cdot)$ at any scale does not differ between NS and SV patients; Conversely, the change in $C_{3}^{(1,0.5)}(j=7)$ indicates a strong positive asymmetry of the fluctuations of $\ln L^{(p, \gamma)}(j, \cdot)$ for NS patients. In equivalent terms, the fluctuations of the time series of NS patients are characterized by the same variance but stronger power law like heavy tails and stronger departure from log-normal tails as those of SV patients.

\section{Conclusions}

Making use of the recently proposed p-leader wavelet coefficients multifractal formalism, the multifractal and multiscale properties of RR interarrival HRV time series temporal dynamics of survivor and non-survivor congestion heart failure patients were compared. For both classes of patients, clear scaling and multifractal properties are evidenced. While a (weakly) significant difference is observed for the selfsimilar scaling exponent $c_{1}^{(1,0.5)}$, no clear differences are evidenced for the multifractal exponents $c_{2}^{(1,0.5)}$ and $c_{3}^{(1,0.5)}$.

Instead, clear differences are reported for the first and third log-cumulant functions, $C_{1}^{(1,0.5)}$ and $C_{3}^{(1,0.5)}$, for scale $A=2^{7} \simeq 100$ beats. Equivalent results are obtained with scales $2^{6}$ or $2^{8}$ corresponding to $\simeq 60$ or $\simeq 250$ beats. These results are strongly consistent with (and slightly outperform) those reported in [3] where a significant departure from Gaussianity for detrended increments of the RR interarrival HRV time series is found around a scale of 40 beats.

It comes as a surprising result that the third log-cumulant function yields significant differences when the second one does not, while they are both related to the non-Gaussianity of the data. The differences of the third log-cumulant indicate a subtle change in the heavy tails of the fluctuations that will be further investigated. This preliminary analysis will be further continued by comparing against age-sex matched healthy subjects. Also, the $24 \mathrm{~h}$ data enables us to study potential differences between awake and sleep phases.

\section{REFERENCES}

[1] J. Nolan et al., "Prospective study of heart rate variability and mortality in chronic heart failure : Results of the united kingdom heart failure evaluation and assessment of risk trial," Circulation, vol. 98, pp. 15101516, 1998.

[2] T. Makikallio et al., "Fractal analysis and time- and frequency-domain measures of heart rate variability as predictors of mortality in patients with heart failure," Am. J. Cardiol., vol. 87, pp. 178-182, 2001.

[3] K. Kiyono et al., "Non-Gaussian heart rate as an independent predictor of mortality in patients with chronic heart failure," Heart Rhythm, vol. 5, pp. 261-268, 2008.

[4] S. Akselrod et al., "Power spectrum analysis of heart rate fluctuation: a quantitative probe of beat-to-beat cardiovascular control," Science, vol. 213, no. 4504, pp. 220-222, 1981.

[5] D. Bansal, M. Khan, and A.K. Salhan, "A review of measurement and analysis of heart rate variability," IEEE Electron. \& Commun., pp. 243-246, 2009

[6] A. Rajendra et al., "Heart rate variability: a review," Med Biol Eng Comput., vol. 44(12), no. 12, pp. 1031-51, 2006.

[7] M. Kobayashi and T. Musha, "l/f Fluctuation of heartbeat period," IEEE Trans. Biomed. Eng., vol. BME-29, pp. 456-457, 1982.

[8] C.K. Peng et al., "Long-range autocorrelations and non-Gaussian behavior of the heartbeat," Phys. Rev. Lett., vol. 70, pp. 1343-1346, 1993.

[9] Y. Yamamoto and R.L. Hughson, "On the fractal nature of heart rate variability in humans: effects of data length and $\beta$-adrenergic blockade," Am. J. Physiol., vol. 266, no. 1, pp. R40-R49, 1994.

[10] C.K. Peng, S. Havlin, H.E. Stanley, and A.L. Goldberger, "Quantification of scaling exponents and crossover phenomena in nonstationary heartbeat time series," Chaos, vol. 5, pp. 82-87, 1995.

[11] P.C. Ivanov et al., "Multifractality in human heart rate dynamics," Nature, vol. 399, pp. 461-465, 1999.

[12] L.A.N. Amaral et al., "Behavioral-independent features of complex heartbeat dynamics," Phys. Rev. Lett., vol. 86, pp. 6026-6029, 2001.

[13] K. Kiyono et al., "Critical scale-invariance in healthy human heart rate," Phys. Rev. Lett., vol. 93, pp. 178103, 2004.

[14] K. Kiyono, Z.R. Struzik, N. Aoyagi, and Y. Yamamoto, "Multiscale probability density function analysis: Non-Gaussian and scale-invariant fluctuations of healthy human heart rate," IEEE Trans. Biomed. Eng., vol. 53, pp. 95-102, 2006.

[15] J. Hayano et al., "Increased non-Gaussianity of heart rate variability predicts cardiac mortality after an acute myocardial infarction," Front. Physiol., vol. 2, pp. 65, 2011.

[16] R. Leonarduzzi et al., "Extending multifractal analysis to negative regularity: p-exponents and p-leaders," in IEEE Int. Conf. Acoust., Speech, and Signal Proc., Florence, Italy, May 2014.

[17] R Leonarduzzi et al., "p-exponent and p-leaders," 2014, in preparation.

[18] S. Jaffard and C. Melot, "Wavelet analysis of fractal boundaries," Comm. Math.Phys., vol. 258, no. 3, pp. 513-565, 2005.

[19] S. Jaffard, "Wavelet techniques in multifractal analysis," in Fractal Geometry and Applications: A Jubilee of Benoît Mandelbrot, M. Lapidus et M. van Frankenhuijsen Eds., Proceedings of Symposia in Pure Mathematics. 2004, vol. 72(2), pp. 91-152, AMS.

[20] R. Lopes and N. Betrouni, "Fractal and multifractal analysis: A review," Medical Image analyis, vol. 13: 634-49, 2009.

[21] M. Doret et al., "Multifractal analysis of fetal heart rate variability in fetuses with and without severe acidosis during labor," $A m$. J. Perinatol., vol. 28, no. 4, pp. 259-266, 2011.

[22] S. Jaffard, P. Abry, and H. Wendt, Irregularities and Scaling in Signal and Image Processing: Multifractal Analysis, in Benoit Mandelbrot: A Life in Many Dimensions, M. Frame, ed., World scientific publishing, 2014, to appear.

[23] J.F. Muzy, E. Bacry, and A. Arneodo, "The multifractal formalism revisited with wavelets," Int. J. of Bifurcation and Chaos, vol. 4, pp. 245-302, 1994.

[24] H. Wendt, P. Abry, and S. Jaffard, "Bootstrap for empirical multifractal analysis," IEEE Signal Proc. Mag., vol. 24, no. 4, pp. 38-48, 2007.

[25] S. Mallat, A Wavelet Tour of Signal Processing, Academic Press, San Diego, CA, 1998.

[26] B. Castaing, Y. Gagne, and M. Marchand, "Log-similarity for turbulent flows," Physica D, vol. 68, pp. 387-400, 1993. 